\title{
Research on the Strategic Model for the Translation of Taoist Classics Based on Adaptation Theory
}

\author{
LIU Ze-lin \\ Bozhou University, Bozhou, China
}

\begin{abstract}
The core view of the adaptation theory is that language has a systematic propagation law, and language translation should be unfolded and extended according to the basic structure of the system. This paper has conducted an in-depth study on the translation of classics of the Taoist culture, with the purpose of improving the accuracy of the translation strategy. This paper also focuses on adaptation theory and taking the continuation and adaptation process of discourse as the core of the research, and conducts the construction analysis on the strategic model. The content of this paper is predicted to offer some simple translation reference ideas for professional translators who are dedicated to the direction of classics.
\end{abstract}

Keywords: adaptation theory, Taoist culture, classics translation, strategic model

\section{Introduction}

In the field of linguistics research, language formation and communication process are in a process of constant change. Therefore, most scholars believe that the main reason why people can communicate smoothly is that people can choose language patterns through their own consciousness (Xiao, 2016). In some cases, people can also make up conversions with others through the subconsciousness or directly under an unconscious state. However, most of the time, people may reply to the appropriate statements only through their own inertial positioning. The intrinsic form of this kind of language communication has become the adaptation theory in linguistics (Huang, 2014a). Therefore, in linguistics translation which requires high on specialty, some translators often apply the adaptation theory to carry out translation, with the purpose of promoting the context of classics translation and the specialty characteristics of the language. Therefore, from the character of adaptation theory, the application of adaptation theory as translation theory during the process of Taoist culture classic translation obviously has practical applicative values.

Taoist culture is an extremely important part in Chinese history and culture. Because of its professional educational value and historical and cultural values, Taoist culture books led by the Tao Teh King have been translated by many countries and introduced into other countries' cultural education. However, before the introduction of Taoist culture into other countries, if our people want to ensure that the connotation of Taoist culture can be truly understood by foreigners with the correct perspective and connotation, the translation strategies should be formulated in a more meticulous manner in English translation work, so as to in turn ensure the practical utility of cultural communication. In view of this, this paper takes the adaptation theory as the core 
and conducts fully research on the strategic model of Taoism translation. The purpose is to sum up a set of truly effective strategies for Taoism translation through the adaptation theory.

\section{Clarification of the Main Points of Cultural Classics Translation and the Concept of Adaptation Theory}

\section{Analysis of Conceptual Implications of Adaptation Theory}

Linguistic researchers in the international field often define the adaptation theory as the core of the four points, i.e., context, language, dynamic condition, and degree of consciousness (Lin, 2015a). Among the four points, the context and the adaptation of language all belong to the conceptual scope of the adaptation theory and are the translation points that the classics translators are required to pay special attention to in the process of translation. The important point of the theory "dynamic condition" lies in that it emphasizes that both language communication process and translation process belong to a dynamic interaction. Even if it's under the relatively fixed status of such text as classics, translation thinking and translation behavior also require a certain active characteristic (Bai, 2015). In terms of "degree of consciousness", its meaning lies in that if the people are required to respect their own ideology during the process of translation and communication, it requires the ability of appropriately mobilizing self-consciousness and various language patterns.

\section{The Main Points of Cultural Classics Translation}

Classics refer to ancient cultural books, and in the field of translation, classics also refer to the literature possessing professional cultural values in ancient times. Not all ancient books can be called "classics”. In China, only literatures related to great litterateurs, or medicine or politics can be called "classics” (Xu, 2015). So, from the above positioning, classics have complete academic and cultural values. Therefore, translators must respect three main points in the process of translating cultural classics. First, when translating the classics, we must respect the vocabulary and cultural connotations in the articles and must not abandon the historical and cultural connotations contained in the original text in order to improve the reading fluency. Second, the translator needs to properly quote the cultural connotation of other countries on the basis of the original text. However, the overall style of writing needs to be based entirely on Taoism and cannot deviate from the theme (Lin, 2015b). Thirdly, the translators must read the original text before carrying out the translation and must perform the translation on the premise that they can read or understand the original text. If above three translation points can be effectively applied, the quality of Taoism can be greatly improved.

\section{Analysis of the Basic Forms of Taoism Translation Based on Adaptation Theory}

\section{With Context as the Adaptation Core}

Linguistic context form. In the context of English, context often refers to the context released by the language, while the translation of classics with context as the core of adaptation is required to focus on the scope within the context of dealing with linguistic context (Meng, 2017). In the Taoism, the character "Dao" appears with a very high probability. For example, "The truth dictated is not eternal truth", etc., are all frequently-cited philosophical vocabulary in Taoism. From the surface meaning of the Chinese characters, these characters are almost the same. If a foreigner reads the original text, the thinking in his/her mind will be inevitably disturbed and he/she will be puzzled about the meaning of Chinese character "Tao" (Lai, 2017). Therefore, the job of translators is to present the linguistic context in which different words and characters 
appear in the original text in the mode of adaptation. The translation strategy for linguistic context is mainly "direct interpretation" (Huang, 2014b). "Dao" can be translated into "road" and "ideas", and it can also be directly written as "tao", preserving the characteristics of Taoism. This translation method cannot only highly enhance the foreigners' understanding of Chinese Taoist culture, but also can deepen the actual effect of cultural output by retaining original Taoist cultural characteristics.

Communicative context form. The communicative contexts appeared in Taoism are mostly the content related to geographical environment, spatial relations, time relations, and so on. For readers who use non-Chinese language, with regards to the content of classics completely based on Chinese ancient, if the translators insist on the use of direct translation, the readers' thinking will be disturbed and they will be puzzled, resulting in the failure of completely understanding the philosophical thought delivered by the classics (Wei, 2014). Therefore, when translating the communicative context by using the adaptation theory, the translator should try to apply the general translation method and the alternative translation method as far as possible, so as to make the readers understand the core meaning of Taoism under the geographical knowledge background of their own (Zhang, 2017). For example, in some Taoism, there are often text and paragraphs for introducing the places and surrounding environmental conditions. These paragraphs usually focus on the introduction of the basic forms and development history of Taoist culture. Therefore, translators can use alternative translation method, for example, to present the name meanings of some place in a manner more closely to Western thinking (Cao, 2017). In turn, foreign readers may generate analogical thinking during their reading, which enables them to more profoundly understand the meaning of place names featuring Taoist culture. Figure 1 shows the basic morphological model of Taoism translation strategy.

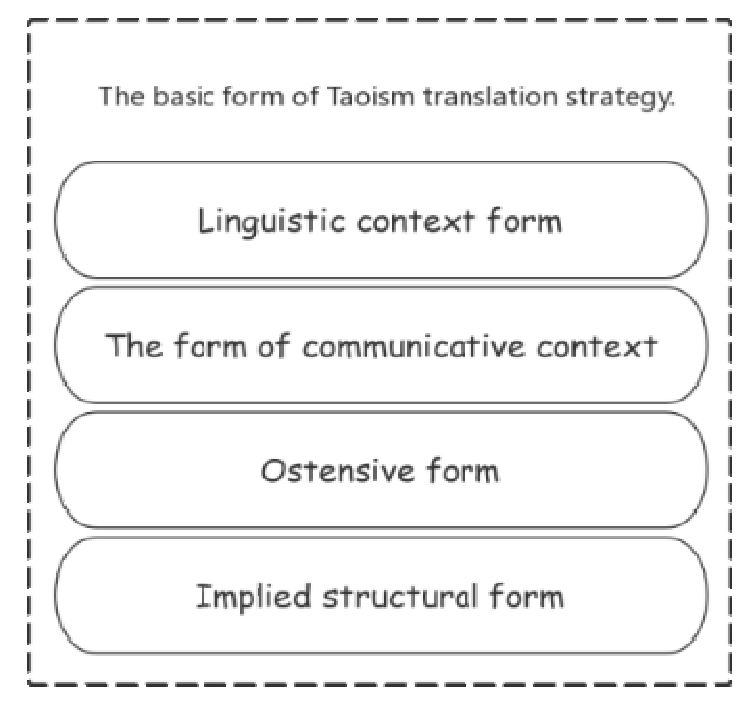

Figure 1. The basic morphological model of Taoism translation strategy.

\section{With Language Structure as the Adaptation Core}

Explicit structure form. The explicit adaptation structure form actually refers to direct translation, and however, the direct translation narrated here is not limited to the meaning of the sentence. The translator may, under the premise of moderate sentence connotation depth, with regard to the word order and grammar of original sentence, carry out the direct translation, so as to retain the practical implications expressed by Taoism 
in "original taste and flavour" (Ni, 2017). However, when the meaning of the sentence and contents expressed are relatively complicated, the translators may also use the word order of the original text, and on the basis of description of original text, explain the specific meanings of each sentence. For example, when translating the "Nine Genres \& Ten Schools", the translators may translate the specific meanings and content of "Nine Genres" and "Ten Schools" separately, through which, readers may understand the cultural connotation more deeply through the explicit structure form provided by the translators, while accepting the phrase of "Nine Genres \& Ten Schools" (Li, 2017). Whether or not the specific explicit structure has practical translation values, the translator can verify the translation result by means of machine translation after the translation work is finished. The specific algorithm formula can be described by using a log-linear model. First of all, the technician should give a formula for source language sentence definition, namely:

$$
\hat{r}(h)=\arg _{r} \max Q(r / h)=\arg _{r} \max \prod_{i=1}^{o}\left[\vartheta_{i}(r, h)\right]^{\chi_{i}}
$$

wherein, we use " $\vartheta_{i}(r, h)$ " to represent the characteristic function of accuracy. Secondly, the technician needs to add the weighting coefficient in the algorithm, and then use the above formula to extract the logarithm, so as to get the translation model, i.e.,

$$
\hat{r}(h)=\arg _{r} \max \sum_{i=1}^{o} \chi_{i} s_{i}(r, h) .
$$

when the translator needs to check the translation result, it only needs to add the specific original text and translation text in the translation system, and then match the probability through above formula. The calculated probability value is the reference content for determining the correctness of translation results.

Implicit structure form. There are obvious differences between the implicit structural form and the explicit structural form, i.e., the translation generated with implicit structural form as the core has already disorganized the word order form of original text. Therefore, when the translators apply this thinking to do translation, they should firstly sort out the thinking and word order of the original text when carrying out the translation by using this thinking, and then rearrange the order of the translation text according the actual order of the content.

\section{Analysis on the Particularity of the Taoism Translation Based on Adaptation Theory}

First, the dynamic characteristics mostly refer to the change features of the culture and the language. That is, in the contemporary era, language upgrades have been completed in either English or Chinese (Yang, 2017). Therefore, in case of Taoism translation, the translators cannot use old English vocabulary to carry out the translation. It requires to use the modern English and modern English grammar as much as possible, so as to guarantee the readers' reading experience. Second, highlight the cultural consciousness. During the process of highlighting the cultural consciousness, translators must firstly understand the original text that needs translation. The translators must be clear about the central theme that Taoism wants to convey, so as to ensure that the translation results also have real literary values. Figure 2 is a special analytical model of Taoism translation strategy. 


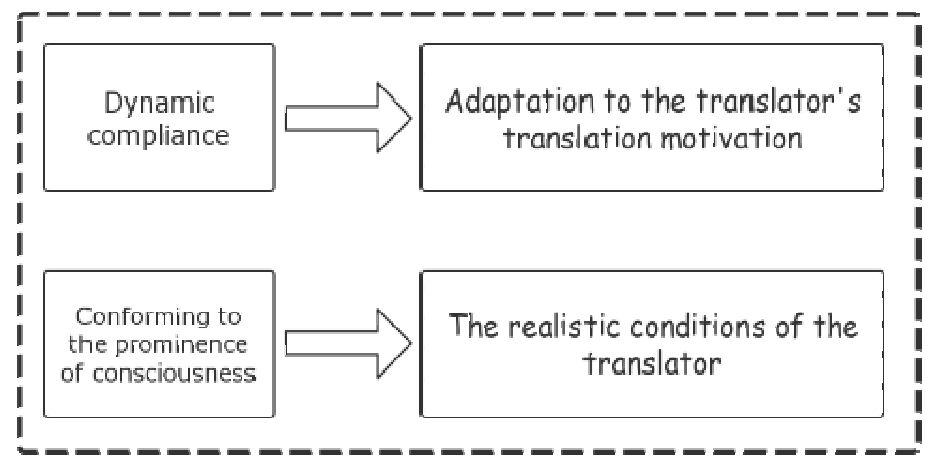

Figure 2. A special analytical model of Taoism translation strategy.

\section{Conclusions}

The translation of Taoism can be regarded as a core way for conveying the Tao of sages. Therefore, when doing translation, translators should use the adaptation theory to carry out the translation. They must respect and understand the original text, and be good at activating the translation ideas, so we can stabilize our real communication value of our country at the level of cultural communication.

\section{References}

Bai, X. Y. (2015). Translation of Chinese classics in the view of national cultural security-an example of the translation of religious culture loaded words in a dream of Red Mansions as an example. Jilin Institute of Education (First Ten Days), 31(8), 134-136.

Cao, L. M. (2017). A study of Chinese discourse in English translation of classics—-taking Pearl S. Buck's English translation of Water Margin as an example. Foreign Language Teaching, 38(4), 89-92.

Huang, H. X. (2014a). On the cultural heterozygosity in the translation of the classics and the self-construction of the translator - an example of the experience of the English translation of the art of war of Sun Tzu as an example of. Crazy English (Teacher Edition), (4), 165-171, 227.

Huang, H. X. (2014b). The context reconstruction and cultural misreading of English translation in the context of high and low cultural contexts-an example of the context reconstruction of Mair English version of Sun Tzu's art of war Nine Discourse. Journal of Northeast Agricultural University (Social Science Edition), 12(2), 59-69.

Lai, Y. H. (2017). A study on the strategy of Chinese classics translation in the context of cross culture-an example of the English translation of a dream of Red Mansions as an example. Jiangxi Social Sciences, 32(12), 239-243.

Lin, Y. B. (2015a). Get out of the "text context"-some thoughts on the translation of classics in the era of fragmented reading. Shanghai Translation, (1), 20-26.

Lin, Y. B. (2015b). The translation strategy of Chinese classics in the "fragmented reading" era—an example of "near translation" in the English version of the Analects of lysino, as an example. Shanghai Normal University (Philosophy and Social Science Edition), 44(5), 143-152.

Li, N. (2017). The cultural rewriting and adaptation of the translation of Folklore-the translation of folklore culture in the English translation of "Fu Le wisdom" in the English translation of. National Translation, (3), 85-91.

Meng, J. (2017). Translation of classics from the perspective of Cultural Adaptation Theory-take Gu Hongming’s Analects of Confucius as an example. Foreign Language Journal, (3), 104-108.

Ni, L. L. (2017). Translation of figurative sentences in the Analects of Confucius from the perspective of adaptation theory-two English versions of Richard and Gu Hongming for example. Modern Language (Language Research Edition), (10), 152-155.

Wei, W. (2014). Translation of the regularity of the ecological environment: An example of the diachronic study of the English translation of Chinese traditional Chinese classics as an example. Journal of University of South China (Social Science Edition), 15(5), 123-126. 
Xiao, C. Y. (2016). Translation of TCM classics under the perspective of Pragmatic Adaptation Theory—taking the English version of Huangdi Neijing as an example. Global Chinese Medicine, 9(3), 336-338.

$\mathrm{Xu}$, J. (2015). Foreign translation and dissemination of Chinese culture and books—on the evaluation and thinking of the great Chinese library. Teaching Theory and Practice, (3), 13-17, 94.

Yang, Y. (2017). From the perspective of adaptation theory, the similarity of the translation strategies of Chinese and English religious works-taking the Jin Kong Jing and the Bible New Testament as an example. Journal of Chifeng University (Science Education Edition), 3(8), 110-112.

Zhang, H. (2017). English translation of modest and respectful title in ancient Chinese: Adaptation and selection-take Liu Dianjue’s version of Meng Zi as an example. English Plaza, (7), 3-6. 